\title{
Fully proportional actigraphy: A new instrument
}

\author{
WARREN W. TRYON \\ Fordham University, Bronx, New York \\ and \\ ROBERT WILLIAMS \\ Computer Science and Applications, Inc., Shalimar, Florida
}

\begin{abstract}
The relevance of activity measurement is reviewed. Technical information regarding a new, small, lightweight, fully proportional accelerometer-based activity monitor suited for a wide range of wrist, waist, and ankle activity measurements over extended time periods in free-ranging persons is presented. Calibration data demonstrating within- and between-device reliability and validity are presented. Field trial data are presented showing that wrist and waist actigraphs can predict kilocalories of energy expended. The issue of how activity monitors should be validated is discussed. Instrument reliability is distinguished from clinical repeatability. Recommendations are provided to assist investigators with instrument selection.
\end{abstract}

There are many reasons why a high-quality ambulatory activity monitor is desirable. Activity is a major dimension of infant temperament (Buss \& Plomin, 1975, 1984; Goldsmith et al., 1987; Hubert, Wachs, Peters-Martin, \& Gandour, 1982; Kagan \& Snidman, 1991; Thomas \& Chess, 1977; Thomas, Chess, \& Birch, 1968; Thomas, Chess, Birch, Hertzig, \& Korn, 1963; Zuckerman, 1991). Genetic influences on activity level during infancy and early childhood are consistently reported (Zuckerman, 1991, pp. 111-116).

The volume edited by Halverson, Kohnstamm, and Martin (1994) documents the development of adult personality from infant and childhood temperament; in this development, activity is both a theoretical and an empirical facet of extraversion, which is one of the "big five" personality factors (Costa \& McCrae, 1980, 1992, 1994, 1995; Digman, 1990, 1994; Goldberg \& Rosolack, 1994; McCrae \& John, 1992; Ozer \& Reise, 1994; Zuckerman, 1991, pp. 28-29). While debate continues regarding the exact number of factors that best characterize adult personality, all investigators recognize extraversion as one of two empirically verified personality traits-neuroticism being the other (Eysenck, 1994). The ability to remain active becomes increasingly important in later life (Tryon, in press-b).

R.W. is an engineer employed by Computer Science and Applications, Inc. He designed and constructed the actigraph reviewed above but does not directly profit from its sale. W.T. is professor of psychology at Fordham University and specializes in activity measurement. He served as an outside evaluator and does not profit from the sale of these actigraphs. While all decisions were mutual, W.T. bears primary responsibility for directing the overall evaluation and presentation. The Model 7164 actigraph reviewed in this article can be purchased from Computer Science and Applications, Inc., 2 Clifford Drive, Shalimar, FL 32579. Correspondence concerning this article shoulds be addressed to W. W. Tryon, Department of Psychology, Fordham University, Bronx, NY 10458-5198 (e-mail: wtryon@murray.fordham.edu).
Disorders of childhood are best categorized as internalizing (a child can be withdrawn, anxious, inhibited) versus externalizing (a child can be disruptive, aggressive, hyperactive) problems (Achenbach \& Edelbrock, 1978; Achenbach, Verhulst, Baron, \& Althaus, 1987; Hinshaw, 1987). Nearly all studies capable of detecting hyperactivity have found such a factor (Hinshaw, 1987). Tryon (1993) has theoretically explained the connection between childhood hyperactivity and the disruptive social/parental relationships that these children also have. Hinshaw (1992) found that external behavior disorders are more predictive of academic underachievement than internal behavior disorders are. Internalizing children are probably less active than externalizing children, but no published evidence bearing on this hypothesis could be located.

Tryon (1991a, 1991b, in press-a) documents that activity is among the inclusion and exclusion criteria of many $D S M$ disorders. Several sleep disorders also have important activity components. Tryon (1991a, pp. 197-204) discusses drug-induced activity effects.

Chronobiologists study activity/rest patterns. Redeker, Mason, Wykpisz, Glica, and Miner (1994) and Redeker, Mason, Wykpisz, and Glica (1995) have used actigraphs to track the return of normal circadian rhythms subsequent to major surgery.

Activity is part of the energy equation governing body weight (Tryon, 1991a, pp. 123-147). Inactivity contributes to coronary heart disease, diabetes mellitus, hypertension, osteoporosis (Siscovick, LaPorte, \& Newman, 1985), and colon cancer (Gerhardsson, Norell, Kiviranta, Pedersen, \& Ahlbom, 1986; Slattery, Schumacher, Smith, West, \& Abd-Elghany, 1988). Activity also reduces the risk of all cause mortality (Tryon, 1991a, pp. 211-213). Several chronic diseases (chronic obstructive pulmonary disease, coronary heart disease) diminish activity level (Tryon, 1991a, pp. 209-220). 
In sum, activity measurement is broadly relevant to infant, child, and adult personality, childhood and adult psychopathology, chronobiology, recovery from surgery, weight control, health risk management, and epidemiology. Activity is suited especially well for measurement by instruments, and there are many reasons why it is more desirable to measure activity than to rate activity (Freedson, 1991; LaPorte, Montoye, \& Caspersen, 1985; Mason \& Redeker, 1993; Melanson \& Freedson, 1996; Montoye \& Taylor, 1984; Tryon, 1985, 1991a). A common deficiency of all but one previous device (Actillume; Ambulatory Monitoring, Ardsley, NY) is that the data obtained are not fully proportional to activity intensity. For example, pedometers do not distinguish between a step taken while walking and one taken while running (unless stride length compensations are made). Tilt sensors do not distinguish slow movements from fast movements. Devices that measure time above threshold distinguish sub- from suprathreshold activity but cannot distinguish within either class of behaviors. The Actillume is the only previous device that contains an 8-bit analog-to-digital (A/D) converter and can distinguish among $2^{8}=256$ levels of activity intensity (128 levels of positive and negative acceleration).

The purpose of the present article is to describe a new fully proportional, small, lightweight, cost-effective activity monitor that can be worn on the wrist, waist, or ankle, has a long battery life, and can continuously collect data every minute of the day and night for 22 days before its memory is completely filled.

\section{ENGINEERING SPECIFICATIONS}

The Computer Science and Applications Model 7164 was designed in 1993. It measures $5.1 \times 3.8 \times 1.5 \mathrm{~cm}$ $(2.0 \times 1.5 \times 0.6 \mathrm{in}$.$) and is lightweight (42.6 \mathrm{~g})(1.5 \mathrm{oz})$. It is housed in a plastic case with "ears" that allow it to be strapped to the wrist or ankle with a Velcro band (see Figure 1). It can also be worn at the waist by inserting it into a snug pouch with a Velcro closure and a belt loop.

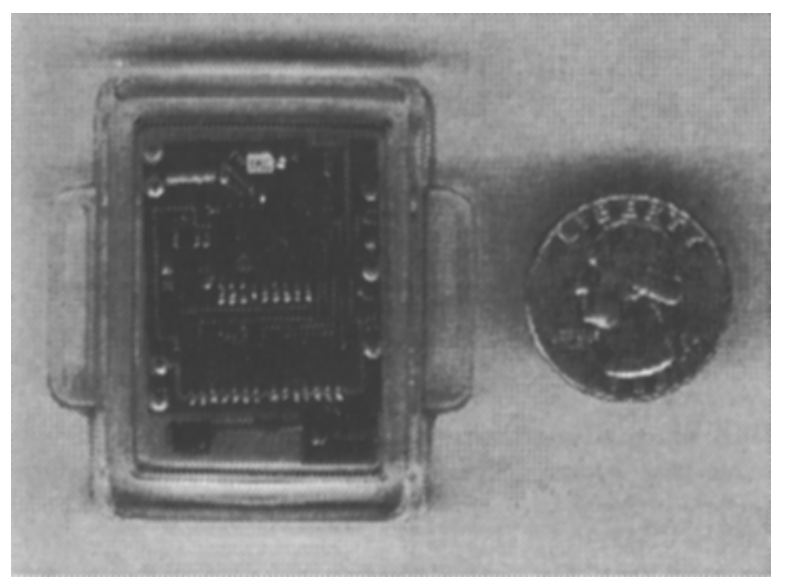

Figure 1. Picture of the CSA Model 7164 actigraph.

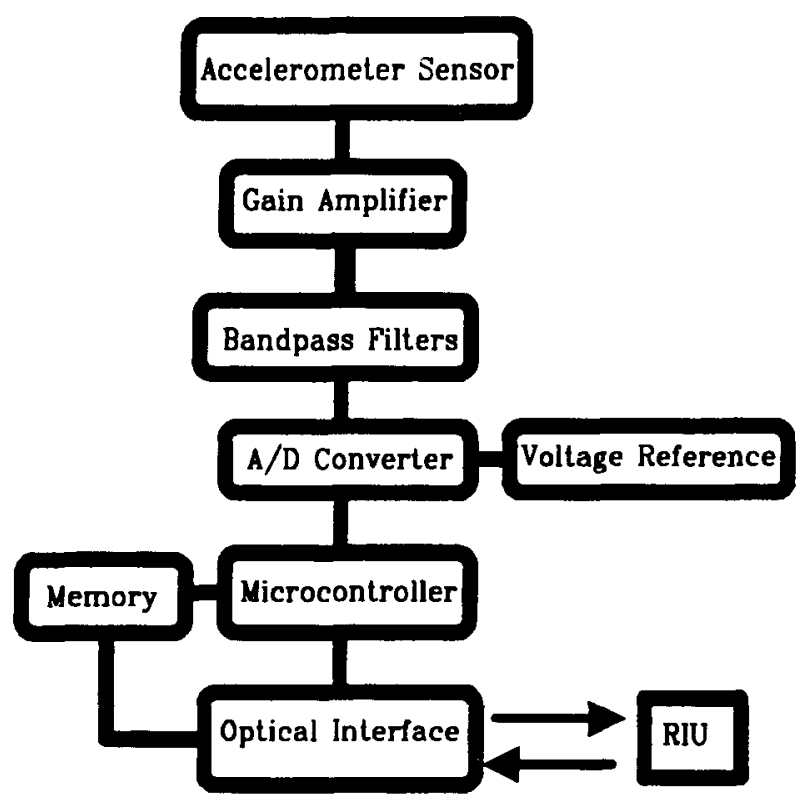

Figure 2. Block diagram of the CSA Model 7164 actigraph. $A / D$, analog to digital; RIU, reader interface unit.

A block diagram of this device is presented as Figure 2 . A cantilevered arm with an attached $1.5-\mathrm{g}$ mass that constitutes the accelerometer sensor can be seen at the bottom of Figure 1. The accelerometer sensor generates a charge that is proportional to the strain imparted to the piezoceramic cantilever beam. The strain is a result of the bending moment caused by acceleration acting on the $1.5 \mathrm{-g}$ mass attached to the free end of the cantilevered beam. The signal produced is conditioned by a charge amplifier and filtered by highpass and lowpass filters. The signal is then digitized at $10 \mathrm{~Hz}$ by an 8 -bit A/D converter and numerically integrated by the microcontroller. The half magnitude points for the lower and upper cutoff frequencies are 0.21 and $2.28 \mathrm{~Hz}$, respectively. Note that these points are simply the frequencies at which a signal of constant magnitude is reduced in amplitude by $50 \%$. Signals outside the passband are attenuated by the filters; however, a $1-g$ acceleration $\left(1 \mathrm{~g}=9.80616 \mathrm{~m} / \mathrm{sec}^{2}\right.$ at sea level at $45^{\circ}$ latitude) with a frequency as low as $0.1 \mathrm{~Hz}$ or as high as $3.6 \mathrm{~Hz}$ is barely detectable, owing to the large dynamic range of the $A / D$ converter. One can think of the filter acting on the signal as a weighting function. The filter applies greater weighting to signals inside the passband than to those outside the passband.

The following two sections indicate that this activity monitor responds to both the intensity and the frequency of movement.

\section{Movement Intensity}

The 8-bit $\mathrm{A} / \mathrm{D}$ converter is capable of discriminating $2^{8}=256$ levels of activity. Because acceleration is positive when the device moves toward the earth (down) and negative when it moves away from the earth (up), zero 
acceleration (no movement) is associated with the center of the A/D scale $(A / D=256 / 2=128)$. This is achieved by biasing the amplifiers so that when no signal is present, a midscale voltage is applied to the A/D converter.

Positive or negative acceleration causes the signal to depart from the midscale value in proportion to the vector component of acceleration. Software calculates the difference between the measured A/D reading and the central value of 128 , thereby removing the sign and retaining only the magnitude of acceleration. The Model 7164 is capable of measuring as little as $5 / 100 \mathrm{~g}$. It responds up to $2.13 \mathrm{~g}$ within a frequency range of $0.1-3.6 \mathrm{~Hz}$. Gain can be adjusted after manufacture to increase or decrease the sensitivity. The passband is set by fixed components and cannot be changed without component substitution.

The internal amplifier is set so that full scale equals $2.13 \mathrm{~g}$. Greater positive accelerations will all equal A/D readings of 256 , and greater negative accelerations will all equal $A / D$ readings of 0 . Dividing $2.13 \mathrm{~g}$ by 128 yields a sensitivity of $0.01664 \mathrm{~g} / \mathrm{bit}$ on the $\mathrm{A} / \mathrm{D}$ converter. There is a deadband of two $( \pm 1) \mathrm{A} / \mathrm{D}$ counts at midscale, thereby causing a threshold of $2(0.1664)=0.033 \mathrm{~g}$. Greater sensitivity and a lower threshold can be achieved by increasing the amplifier gain at the price of reduced dynamic range. If full scale is reduced to $2.0 \mathrm{~g}, \mathrm{~A} / \mathrm{D}$ sensitivity increases to $2.00 / 128=0.0156 \mathrm{~g} / \mathrm{bit}$ and the threshold reduces to $2(0.0156)=0.031 \mathrm{~g}$. Reducing full scale to $1.5 \mathrm{~g}$ further increases A/D sensitivity to $1.5 / 128=0.0117$ $\mathrm{g} / \mathrm{bit}$ and decreases threshold to $2(0.0117)=0.023 \mathrm{~g}$. Further full-scale reduction to $1 \mathrm{~g}$ increases $\mathrm{A} / \mathrm{D}$ sensitivity to $0.0078 \mathrm{~g} /$ bit and reduces threshold to $2(0.0078)=$ $0.016 \mathrm{~g}$. Investigators who use ranges other than the standard plus or minus $2.13 \mathrm{~g}$ should report this fact when presenting their data.

\section{Movement Frequency}

The activity counts registered depend on the frequency as well as intensity of movement. Walking results in vertical (up and down) movements of the waist plus oscillatory movements of the wrists and ankles. The output of the accelerometer is sampled 10 times each second $(10 \mathrm{~Hz})$, and the resulting values are summed over the user-selected epoch. Using a 1-min epoch means that $10 \times 60=600$ measurements are taken and summed each minute, with the minimum possible value being 600 $\times 0=0$. The maximum possible value depends on movement frequency, as described in Table 1 . The 0.1 - to 3.6-Hz passband filter, used to discriminate human movement from vibration and other artifacts, is most transparent at $0.75 \mathrm{~Hz}$, where a pure sinusoid of $2.13 \mathrm{~g}$ results in 46,000 counts $/ \mathrm{min}$. Total counts/minute decrease symmetrically as frequency components increase or decrease within the passband window, even though the amplitude of a pure sinusoid remains at $2.13 \mathrm{~g}$. Frequency components less than $0.1 \mathrm{~Hz}$ and greater than $3.6 \mathrm{~Hz}$ are greatly attenuated by the filters. When the Model 7164 is manufactured, care is taken to adjust each device so that the area in the gain-bandwidth curves are the same. In practice,
Table 1

Activity Counts/Minute Associated With 2.13-g Sinusoidal Movements Varying From 0.1 to $10.0 \mathrm{~Hz}$

\begin{tabular}{ccc}
\hline $\mathrm{Hz}$ & Activity Counts/Min & \multicolumn{1}{c}{ Comments } \\
\hline 0.10 & 11,500 & \\
0.21 & 23,000 & Half magnitude $(-6 \mathrm{~dB})$ \\
0.29 & 32,200 & Half power $(-3 \mathrm{~dB})$ \\
0.50 & 43,700 & \\
0.75 & 46,000 & Signal peak for $2.13 \mathrm{~g}$ \\
1.00 & 43,700 & \\
1.25 & 39,800 & \\
1.50 & 35,300 & \\
1.66 & 32,200 & \\
1.75 & 30,700 & \\
2.00 & 26,800 & \\
2.28 & 23,000 & \\
2.50 & 19,900 & \\
2.75 & 17,600 & \\
3.00 & 15,300 & \\
3.58 & 11,500 & \\
4.00 & 9,200 & \\
5.00 & 6,100 & \\
6.00 & 5,000 & \\
7.00 & 3,800 & \\
8.00 & 3,100 & \\
9.00 & 2,700 & \\
10.00 & 2,300 & \\
\hline
\end{tabular}

the area under the gain-bandwidth curves of each activity monitor are matched to within 5\% of one another.

\section{Data Capacity}

The present design can accommodate from 8 to $64 \mathrm{~K}$ of nonvolatile RAM. All of the memory is available for data storage. Two bytes of RAM are used to store the value at the end of each epoch. Because there are $1,440 \mathrm{~min}$ per day, 2,880 bytes of RAM are filled each day when 1-min epochs are chosen for the summation period. A Model 7164 can collect data for 22.75 days if one uses 1-min epochs.

One 2430 lithium coin cell battery will power this device for 4-6 months, depending on use. The number of usable hours of battery life remaining is displayed each time the activity monitor is initialized. These batteries can be obtained from sources such as Radio Shack (No. 23-166).

\section{Initializing the Actigraph}

The activity monitor is initialized and data are downloaded by placing it into a reader interface unit (RIU), which is connected to the serial port of an IBM or compatible computer, desktop or portable. All data communications are transmitted through the plastic case using a coded beam of infrared light at 4,800 baud (bits per second). The RIU is powered by a $9-\mathrm{V}$ ac to dc converter that plugs into $120 \mathrm{~V}$ ac. The absence of external switches, jacks, or displays on the Model 7164 makes it psychologically inert as well as dust and water resistant and consequently more dependable.

The activity monitor is initialized by placing it into the $\mathrm{RIU}$ and running a program called RIU.exe. The user is prompted to select a serial port by pressing a " 1 " or " 2 ." 
Table 2

Sample Initialization Menu

Activity Monitor Initialization Routine for Data Collection. This routine will force a reset of the Activity Monitor. Do you wish to continue $(y / n)$ ?

CSA Inc. Activity Monitor

Model 7164

SN:xxx13 V1.2

Battery Life Remaining: 2479 hrs

Read 00:01:00 for Epoch from file EPOCH.CFG

Do you wish to set/change Epoch $(\mathrm{y} / \mathrm{n})$ ?

Activity Monitor Run Time will be 68.2 Hours

Hit Return for Current Time \& Date?

Do you wish to set PC time and or date $(\mathrm{y} / \mathrm{n})$ ?

Do you wish to set/change Start Time \& Date $(\mathrm{y} / \mathrm{n})$ ?

Count down Timer: $102 \mathrm{sec}$

Epoch Timer: $60 \mathrm{sec}$

Activity Monitor Timer Started

Writing Initialization Data to Disk File SNxxx13.INI Finished

Pressing the F9 function key activates an initialization menu, illustrated in Table 2. The serial number of the activity monitor is displayed, along with the number of hours of remaining battery life. The user is informed of the current epoch length, with the option to change it. Run time is then calculated on the basis of the chosen epoch length. The user is informed of the current time and date and allowed to change the computer clock. The user is asked to set or change the start time and date. One would choose "no" if several activity monitors were being programmed to begin collection at the same time. The unit can be programmed to begin data collection at any year, month, day, hour, minute, second that the user chooses. The number of seconds until data collection begins is displayed as the Count Down Timer. The epoch length is confirmed. That the activity monitor timer has started is confirmed, and writing the initialization data to disk is confirmed. Initialization is further confirmed by a periodic triple flash emitted from the activity monitor and reflected in a metal mirror in the RIU. Data collection can be confirmed after the start time and date has elapsed by replacing the activity monitor in the RIU and observing a continuous $10-\mathrm{Hz}$ flashing light. The same confirmation can be obtained in the field by bringing a small magnet to the side of the activity monitor. The magnet supplied by CSA is a cylindrical rod 0.125 in. in diameter by 0.750 in. in length. It is not possible to destroy memory with this magnet.

\section{Downloading Data}

Pressing the F2 function key activates the downloading menu, which is illustrated in Table 3 . The serial number and battery life are displayed along with the current address, which equals the number of data points collected. The data are downloaded at 4800 baud in an NRZ format compatible with standard asynchronous protocol. The ef- fective download rate is approximately 3000 bits per second, owing to protocol overhead of start/stop bits and background addressing of the serial memory. A 64K maximum file size requires $64(1 \mathrm{~K}=1,024) 8 \mathrm{bits} / \mathrm{byte} /$ $3000 \mathrm{bits} / \mathrm{sec}=175 \mathrm{sec}(2 \mathrm{~min}, 55 \mathrm{sec})$ to download. The data are stored in a default file, unless the user provides a new name. It is important that the INI file containing initialization information be available to the downloading program.

The data are written in ASCII format to either a default or user-specified file name. The file header contains the following information: file name, start time, start date, epoch period (hh:mm:ss), download time, download data, current memory address (equal to the number of data points downloaded), and battery life remaining (in hours). The data can be written in several formats. A format.cfg file can be used to specify the format by which the data are written to a disk file. In the most straightforward format, the first column is the epoch number and the second column is the activity integral for that epoch. Options to write the data in $N \times N$ arrays exist to facilitate formats ready for import into popular spreadsheets such as Microsoft's Excel. The data columns are fielded by eight characters, with the largest number occupying seven characters.

\section{CALIBRATION}

If an accelerometer is dc connected, it can be calibrated by the following static method: (1) place its sensitive axis horizontally and adjust the balance control to read zero volts, (2) point the sensitive axis down and measure the positive voltage output associated with $+1 g$, (3) point the sensitive axis $u p$ and measure the negative voltage output associated with $-1 \mathrm{~g}$, and (4) divide the signed voltage differences by $2 g$ to calculate volts per $g$.

The accelerometer in the Model 7164 activity monitor is ac coupled to avoid dc drift caused by temperature variation and other factors, thereby avoiding the necessity of hourly recalibration. Consequently, simple static calibration methods are not appropriate, and a dynamic calibration procedure must be used.

\section{Pendulum}

The pendulum calibration procedure is simple in principle, and the mathematics of a decaying pendulum swing have long been well understood. Tryon (1991a, p. 51) published the results of four pendulum tests on an Ambulatory Monitoring Actillume which also contains an

Table 3

Sample Download Menu

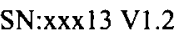

Battery Life Remaining: 2479

Current Address is $\mathbf{4 0 9 6}$

Downloading Data ..

Assign File SNxxx13.dat

Do you wish to use this file $(\mathrm{y} / \mathrm{n})$ ? 
8-bit A/D converter. The result was a smooth decline from a high peak associated with the initial large-amplitude, large-acceleration swings, down through the final lowamplitude, low-acceleration swings. The height of the decay function is proportional to the measured acceleration from its initial maximum to the time at which acceleration drops below the activity monitor's threshold.

The pendulum must be made to be highly repeatable from test to test if variation in measured response is to be entirely attributed to measurement error in the activity monitor. The greatest problem is to control the decay rate of the free swinging pendulum. Even when extreme care is taken in constructing the pendulum fixture, factors such as pivotal friction and air drag can, and do, influence the actual decay. Small variations due to these effects have been tracked with CSA activity monitors, which indirectly testifies to their sensitivity. CSA constructed its pendulum with ball bearings on the pivot (see Figure 3). Air drag and friction of the bearings induce the decay; without drag and friction, the pendulum would swing back to its release point indefinitely. These two parameters can vary in time; therefore, some method of measuring the actual decay of the pendulum must be employed to reliably calibrate activity monitors. One approach is to permanently install a commercial instrumentgrade accelerometer on the pendulum bob with the activity monitor and record actual acceleration during the pendulum tests and/or calibrations.

CSA constructed an instrumentation accelerometer based on the IC Sensors (Milpitas, CA) 3026-002-S solid state piezoresistive bridge. This accelerometer is responsive to $\mathrm{dc}$ and was calibrated by using gravity and various inclination angles to check linearity. After we were satisfied with the accuracy of the solid state accelerometer, we mounted it on the pendulum piggy back with the activity monitor. This arrangement provided a standard reference source for measuring the acceleration and decay of the pendulum while the activity monitor was being tested. Repeated testing with this arrangement (1) confirmed results obtained by the activity monitors and (2) verified variation in pendulum decay. Hence, not all deviations between consecutive pendulum test series can be attributed to actigraph unreliability. Attempts to use a

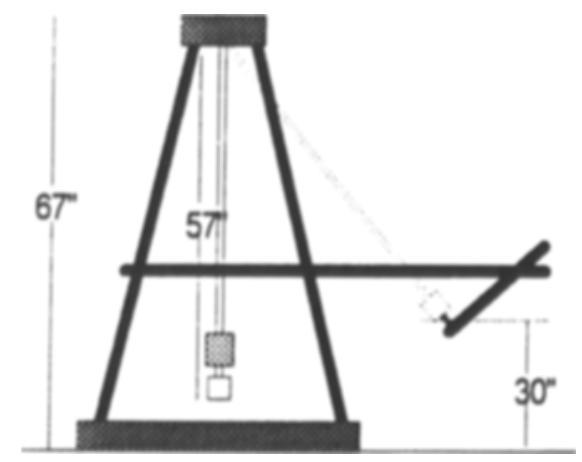

Figure 3. Diagram of the CSA pendulum. free decaying pendulum as a calibration source without some measurement of the actual acceleration and decay entails a small but measurable inconsistency.

Done properly, pendulum data can be used to demonstrate both reliability and validity. The activity monitor is reliable to the extent that repeated tests on identical pendulum calibration runs produce duplicate graphs. It is possible to calculate the pairwise differences for each epoch over the course of the calibration interval across several calibration runs and to calculate the mean difference. For example, given three pendulum tests $(a, b, c)$, each entailing $N 10$-sec epochs, one could calculate the following differences: $a 1-b 1, a 1-c 1, b 1-c 1, a 2-b 2, a 2-c 2$, $\mathrm{b} 2-\mathrm{c} 2, \ldots, \mathrm{a} N-\mathrm{b} N, \mathrm{a} N-\mathrm{c} N, \mathrm{~b} N-\mathrm{c} N$. The mean and standard deviation of the $3 N$ differences would index the consistency with which the activity monitor measured the same physical phenomenon.

The results of three pendulum tests of the same Model 7164 actigraph are presented in Figure 4. Plot symbols and line types have been omitted, to emphasize the extremely high degree of congruity among the three test results over the large range of activity studied.

Figure 5 displays the $A / D$ count differences $a-b, a-c$, and $b-c$ derived from Figure 4. Plot symbols and line types were again omitted for clarity. Note that the maximum difference is only 30 counts out of 1,208 counts $(2.5 \%)$. This occurs at epoch number six in the $b-c$ series. The mean (and $S D$ ) for the three sets of count differences are: $a-b=6.78(6.45), a-c=5.66(5.48)$, and $b-c=6.68(6.36)$. The largest mean of 6.78 is about half of one percent of 1,208 counts $(0.56 \%)$. All data are presented in Table 4. Some of these differences are due to differences in the rate of pendulum decay across the three trials, and therefore, not all of this variation is due to the activity monitor.

Activity monitor validity can also be determined from pendulum data. The activity monitor is valid if the epoch value generated is, within a linear scale factor, equivalent to the theoretically expected value associated with the temporal midpoint of the epoch in question. Said otherwise, the activity monitor data should decay in the same way as the pendulum amplitude decays. Visual inspection is often sufficient to determine that the instrument output traces a smooth decay function. This can be seen in Figure 4.

The validity of an activity monitor can be determined by comparing its output with a standard reference output associated with pendulum decay to within a constant scale factor. Figure 6 compares the results of a pendulum test with the Model 7164 actigraph with the IC Sensors reference standard attached to the pendulum bob. It can be seen that the deviations are quite small throughout the entire pendulum test, thereby demonstrating the validity of this actigraph. The departure between the two curves is due to the analog filter present in the Model 7164 and absent in the IC Sensors accelerometer. As the pendulum decays, the frequency falls off the peak of the Model 7164, resulting in the lower gain. The activity monitor therefore traces the lower line. 


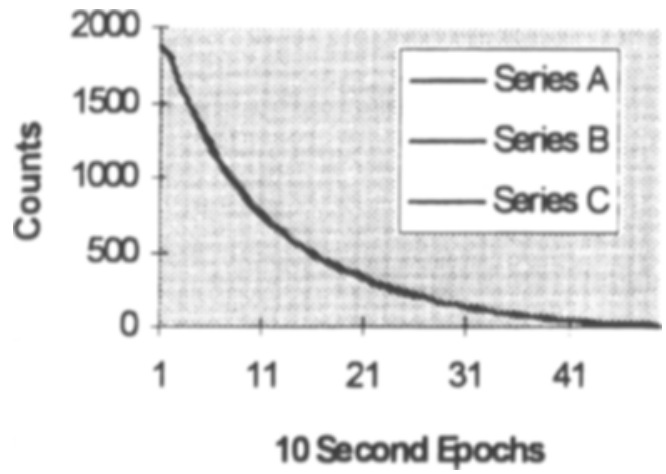

Figure 4. Three pendulum decay series for one actigraph (Serial Number 20). The three data sets trace virtually the same curve.

\section{Spinning}

A problem with the pendulum calibration procedure is that the magnitude of acceleration constantly changes and is a function of the pendulum arc swing and location of the activity monitor on the pendulum rod. Frequency of the free swinging pendulum is for the most part constant; it speeds up slightly as the amplitude decays and is proportional to the square root of the length between the pivot and the bob mass. The greatest problem in obtaining consistent calibration results with a pendulum is control of the decay rate as stated earlier. While the amplitude range, typically $1 / 2 g$, provided by a reasonably sized pendulum may adequately sample the physical range associated with anticipated behaviors, the single frequency (typically $\sim 1 \mathrm{~Hz}$ ) does not sample the frequencies defined by the passband filter $(0.1-3.6 \mathrm{~Hz})$. This problem is solved by spinning the activity monitor at various speeds and possibly angles.

The activity monitors manufactured by CSA are calibrated with a fixture that rotates the monitor in the vertical plane. This spinner, like a car, has a wheel attached to a horizontal-axle drive shaft. Rotation of the axle causes the wheel to spin in the vertical plane. The actigraph is firmly attached to the face of the wheel so that the cantilevered beam points horizontally to the left (see Figure 1). The wheel is rotated counterclockwise. The acceleration sensed by the activity monitor's cantilevered beam mass while attached to the spinner is defined by Equation 1, where $g$ is the earth's gravitational constant at the test site (e.g., $9.80616 \mathrm{~m} / \mathrm{sec}^{2}$ at sea level at $45^{\circ}$ latitude). Phi is the angle that the drive-shaft axle makes with the horizontal plane. Phi is typically set to zero because $\cos (\phi=$ 0 ) is one, thereby removing this term from the equation. Lower acceleration levels can be achieved by tilting the drive shaft. For example, tilting the drive shaft to a $60^{\circ}$ incline produces a maximum acceleration of $0.5 \mathrm{~g}$ because acceleration $=1 \mathrm{~g} \cos (60)=0.5 \mathrm{~g}$. The resulting $g \sin (\theta)$ term reflects primary acceleration. Theta is the instantaneous angle of the piezoceramic beam about the spinning axis and is the product of the angular speed of rotation (omega, $\omega)$ and time $(\theta=\omega t)$. Omega is the spinning rate and is varied from 0.1 to $10.0 \mathrm{~Hz}$ (revolutions per second) $(\omega=d \theta / d t)$. The differential operator $d$ is with respect to time. The radius $r$ is the length of the cantilever beam from the mounting point to the $1.5 \mathrm{-g}$ mass. The axis of rotation is near, but not directly under, the point of attachment. This small displacement is unimportant, because all points on the spinning wheel rotate at the same angular rate, omega. The $r d(\theta / d t)^{2}$ term reflects centrifugal acceleration and acts on a line through the netrual axis of the beam and does not cause any bending. The $r d^{2}(\theta) / d t^{2}$ term reflects the change in acceleration resulting from changes in angular speed due to changes in the spinning rate. Provided that spin frequency is changed slowly, the differential term is swamped by the $g \sin \theta$ term. For example, calibration procedures control the change in angular speed to $5 \mathrm{~Hz}(5 \times 60=300 \mathrm{rpm})$ over $1 \mathrm{~min}$ and result in a spin down acceleration of less than $1 / 1000 \mathrm{~g}$ compared to the first term of $1 \mathrm{~g}$ modulated by $\sin (\theta)$. In calibrating the activity monitors, the effects of spin down acceleration are ignored:

$$
\text { Acceleration }=g \cos \phi \sin \theta+r\left[d^{2} \theta / d t^{2}+(d \theta / d t)^{2}\right] \text {. }
$$

The magnitude of acceleration varies as a sinusoid with a peak magnitude of $\pm 1 \mathrm{~g}$. The output of the accelerometer is sampled at $100 \mathrm{~Hz}$ by the activity monitor's A/D circuit (10 times the normal rate), and the digital values are transmitted to the host computer. Software in the host computer processes these data and calculates the gain bandwidth on the basis of peak-to-peak signal variation and rotational frequency. This method does not require a second channel to measure actual acceleration, because $\pm 1 \mathrm{~g}$ is always the excitation force magnitude and is independent of rotation frequency. The radial and tangential acceleration components associated with this process appear as a dc component if one takes care to limit the rate at which the rotation frequency is changed to $5 \mathrm{~Hz}$ per minute or less, as is the case with current calibration procedure.

The spinner calibration method provides replicable frequency components likely to be encountered in the field

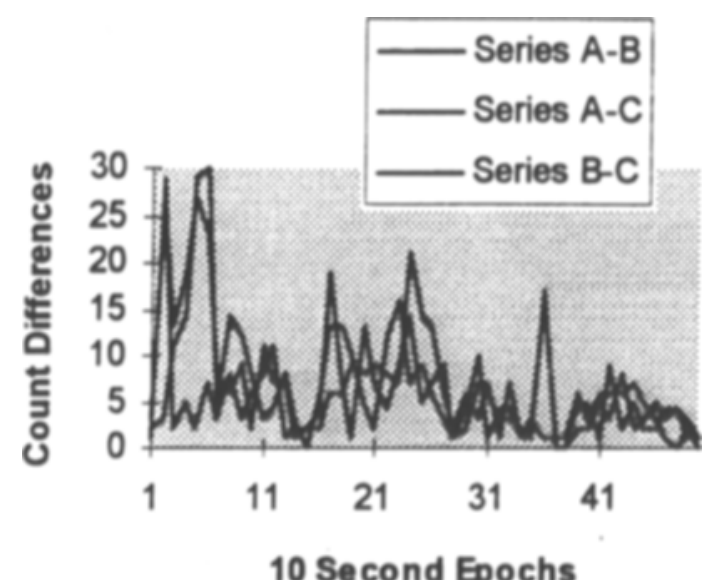

Figure 5. Pendulum decay differences, $A-B, A-C$, and $B-C$, calculated from Figure 4. 
Table 4

Reliability Data Calculated Among Three Tests of the Same Actigraph (Series A, B, \& C).

These Data Were Used to Create Figures 4 and 5

\begin{tabular}{|c|c|c|c|c|c|c|}
\hline Epoch & Counts A & Counts B & Counts $\mathrm{C}$ & $\mathbf{A}-\mathbf{B}$ & $A-C$ & B-C \\
\hline 1 & 1877 & 1874 & 1876 & 3 & 1 & 2 \\
\hline 2 & 1814 & 1788 & 1785 & 26 & 29 & 3 \\
\hline 3 & 1624 & 1611 & 1622 & 13 & 2 & 11 \\
\hline 4 & 1478 & 1459 & 1473 & 19 & 5 & 14 \\
\hline 5 & 1338 & 1311 & 1340 & 27 & 2 & 29 \\
\hline 6 & 1201 & 1178 & 1208 & 23 & 7 & 30 \\
\hline 7 & 1087 & 1079 & 1084 & 8 & 3 & 5 \\
\hline 8 & 986 & 980 & 994 & 6 & 8 & 14 \\
\hline 9 & 912 & 903 & 915 & 9 & 3 & 12 \\
\hline 10 & 819 & 817 & 825 & 2 & 6 & 8 \\
\hline 11 & 758 & 747 & 750 & 11 & 8 & 3 \\
\hline 12 & 691 & 684 & 680 & 7 & 11 & 4 \\
\hline 13 & 628 & 621 & 629 & 7 & 1 & 8 \\
\hline 14 & 572 & 573 & 570 & 1 & 2 & 3 \\
\hline 15 & 536 & 534 & 534 & 2 & 2 & 0 \\
\hline 16 & 481 & 477 & 483 & 4 & 2 & 6 \\
\hline 17 & 457 & 438 & 451 & 19 & 6 & 13 \\
\hline 18 & 415 & 422 & 409 & 7 & 6 & 13 \\
\hline 19 & 372 & 373 & 363 & 1 & 9 & 10 \\
\hline 20 & 360 & 347 & 352 & 13 & 8 & 5 \\
\hline 21 & 338 & 331 & 329 & 7 & 9 & 2 \\
\hline 22 & 287 & 283 & 295 & 4 & 8 & 12 \\
\hline 23 & 268 & 259 & 275 & 9 & 7 & 16 \\
\hline 24 & 260 & 246 & 239 & 14 & 21 & 7 \\
\hline 25 & 227 & 222 & 213 & 5 & 14 & 9 \\
\hline 26 & 210 & 203 & 197 & 7 & 13 & 6 \\
\hline 27 & 198 & 189 & 192 & 9 & 6 & 3 \\
\hline 28 & 166 & 165 & 164 & 1 & 2 & 1 \\
\hline 29 & 150 & 154 & 156 & 4 & 6 & 2 \\
\hline 30 & 140 & 150 & 143 & 10 & 3 & 7 \\
\hline 31 & 128 & 127 & 121 & 1 & 7 & 6 \\
\hline 32 & 116 & 120 & 117 & 4 & 1 & 3 \\
\hline 33 & 101 & 105 & 108 & 4 & 7 & 3 \\
\hline 34 & 93 & 94 & 92 & 1 & 1 & 2 \\
\hline 35 & 86 & 89 & 87 & 3 & 1 & 2 \\
\hline 36 & 67 & 68 & 84 & 1 & 17 & 16 \\
\hline 37 & 68 & 69 & 68 & 1 & 0 & 1 \\
\hline 38 & 62 & 63 & 62 & 1 & 0 & 1 \\
\hline 39 & 55 & 49 & 53 & 6 & 2 & 4 \\
\hline 40 & 49 & 46 & 51 & 3 & 2 & 5 \\
\hline 41 & 39 & 45 & 44 & 6 & 5 & 1 \\
\hline 42 & 39 & 45 & 36 & 6 & 3 & 9 \\
\hline 43 & 25 & 31 & 33 & 6 & 8 & 2 \\
\hline 44 & 31 & 24 & 29 & 7 & 2 & 5 \\
\hline 45 & 26 & 21 & 23 & 5 & 3 & 2 \\
\hline 46 & 17 & 20 & 22 & 3 & 5 & 2 \\
\hline 47 & 16 & 15 & 19 & 1 & 3 & 4 \\
\hline 48 & 15 & 15 & 11 & 0 & 4 & 4 \\
\hline 49 & 10 & 12 & 9 & 2 & 1 & 3 \\
\hline \multirow[t]{3}{*}{50} & 9 & 9 & 10 & 0 & 1 & 1 \\
\hline & & $M$ & & 6.78 & 5.66 & 6.68 \\
\hline & & $S D$ & & 6.45 & 5.48 & 6.36 \\
\hline
\end{tabular}

(e.g., $0.1-10.0 \mathrm{~Hz}$ ). Figure 7 illustrates the graphic output associated with this calibration procedure.

Reliability can be evaluated by repeating the preceding calibration procedure multiple times. The analytic procedures for evaluating pendulum reliability described above can be used here. In this case, the mean and standard deviation of the area under the curve between 0.25 and $2.50 \mathrm{~Hz}$ associated with five repeated tests of a par- ticular Model 7164 (Serial No. 20) had a mean of 1.025 and a standard deviation of 0.007 . The values of 0.25 and $2.50 \mathrm{~Hz}$ were chosen because they encompass a decade change in frequency, lie outside the upper half power point of the filters, and cover the major portion of power passed by the filter. It is coincidental that the results of the $1-g$ spin test integrals are approximately equal to unity for the 2.13-g full-scale gain.

\section{Units of Measure}

The sensor used to detect motion in the Model 7164 is a cantilevered piezoceramic plate that produces a transient electric charge in response to a force acting on a seismic mass attached at the free end of the plate. The reader should note that the charge is transient and bleeds off to zero in steady-state acceleration environments. The sensor can only measure time-varying acceleration, the derivative of acceleration with respect to time, as given by Equation 2 . The second and third terms are made small by experimentally restricting the rate with which spinning changes to $5 \mathrm{~Hz}$ per minute while varying spinning frequencies from 0.1 to $10.0 \mathrm{~Hz}$.

$$
\frac{d A}{d t}=g \omega \cos \phi \cos \omega t+r \frac{d^{2} \omega}{d t^{2}}+2 r \omega \frac{d \omega}{d t} .
$$

This experimental control, along with keeping the drive shaft horizontal so that $\phi$ is zero, makes $\cos \phi=1.0$ and reduces Equation 2 to Equation 3. This equation specifies the third derivative for how the 1.5-g mass changes with respect to time in relation to the anchor point of the cantilevered plate:

$$
\frac{d A}{d t}=g \omega \cos \phi \cos \omega t=g \omega \sin \omega t .
$$

Thus the rate of acceleration is a sinusoidal function of $\omega t$, amplitude modulated by $\omega$, which is the hertz value given in Table 1. Under linear acceleration, $\omega$ becomes zero, Equation 3 becomes zero, and the measured value becomes zero. This does not occur during calibration, because the device is constantly rotating and acceleration is therefore constantly changing. That limbs are attached

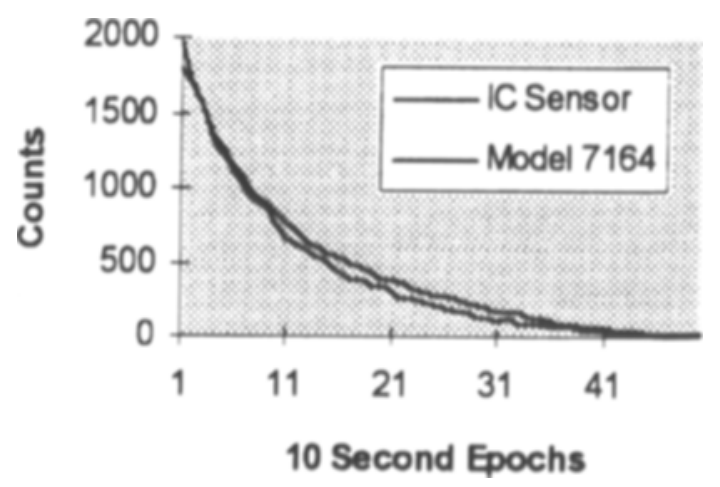

Figure 6. Pendulum test of Model 7164 actigraph with a piggybacked IC Sensors solid state accelerometer. The activity monitor tracks the lower line because of its analog filters. 


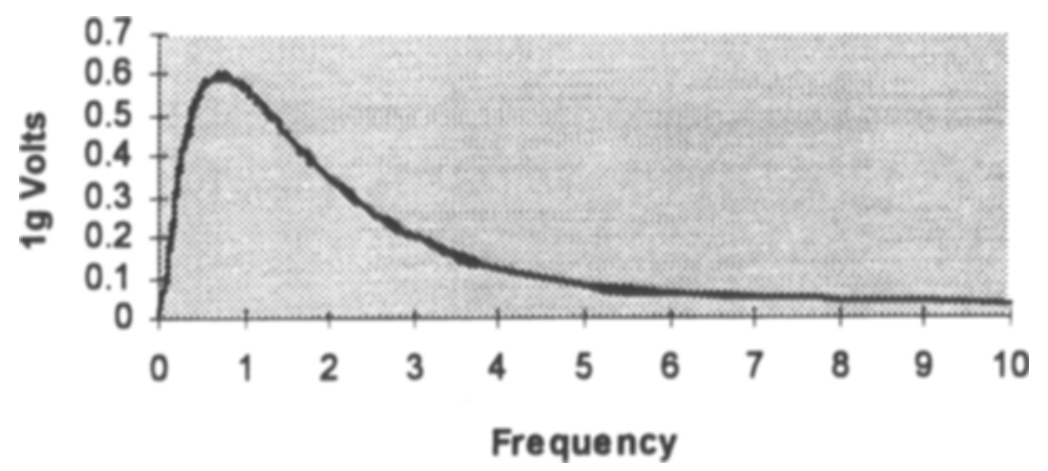

Figure 7. Three runs of the same actigraph undergoing a 1-g spinner series.

to the trunk and that the waist moves in spiral fashion while ambulating prevent this from occurring in the field as well. The instantaneous $\mathrm{A} / \mathrm{D}$ measurement quantifies this third derivative with an instantaneous sampled measurement unit of $g /$ second. The 8 -bit $A / D$ converter discriminates 256 levels, but 128 are of positive acceleration and the other 128 are of negative acceleration. For a full scale of $2.13 \mathrm{~g} / \mathrm{sec}$ per 128 quantized levels, the resolution of the instantaneous measurement is $2.13 \mathrm{~g} / \mathrm{sec} /$ $128 \mathrm{~A} / \mathrm{D}$ counts or $0.01664 \mathrm{~g} / \mathrm{sec} /$ count. Multiplying each measurement by the sample period $(d t=0.1 \mathrm{sec})$ integrates the third derivative, yielding the second derivative with respect to time, or acceleration. This integral is composed of rectangles with a vertical dimension of $\mathrm{g} / \mathrm{sec} /$ count and a horizontal dimension of seconds, resulting in a final measurement unit of $\mathrm{g} /$ count. Multiplying $0.01664 \mathrm{~g} /$ $\mathrm{sec} / \mathrm{count}$ by $0.1 \mathrm{sec}$ yields quantized measurement units of $0.001664 \mathrm{~g} /$ count or $1.664 \mathrm{milli}-\mathrm{g} /$ count.

Unfortunately, one cannot use the $0.001664 \mathrm{~g} /$ count unit to work backward from counts to $g s$ because of the dependency of counts on signal frequency as well as amplitude. Table 1 clearly shows that the activity counts depend critically on the frequency by which acceleration is time varying, and that information is not recorded or utilized by the present device. Future models will include an option to use the frequency information presented in Table 1 to calculate measured units of $g$. Then the integral of acceleration can be calculated off line, resulting in velocity. This advance will allow one to calculate energy expenditure in the physical rather than biological sense, as will be discussed next.

Kinetic energy can be calculated from a knowledge of mass $(m)$ and velocity $(v)$ according to Equation 4 . A person's center of gravity is approximately at the waist, which means that person's body weight in kilograms can be used to estimate $m$ if the actigraph is attached to the waist. The average acceleration acting over the user-defined epoch, say $60 \mathrm{sec}$, results in velocity $v$. Hence, $v$ can be calculated by multiplying the average acceleration by the selected epoch duration in seconds. Kinetic energy can be calculated by squaring this value, multiplying by $m$, and dividing by 2 :

$$
E_{k}=\frac{m v^{2}}{2} \text {. }
$$

The work-energy principle defines work in terms of change in kinetic energy. Hence, calculating and summing consecutive differences in kinetic energy calculations will result in average work performed over two or more userselected epochs. Work and energy are measured in the same units. The joule $(\mathrm{J})$ is the meter-kilogram-second unit for energy and work. One megajoule (1 MJ) equals $239 \mathrm{kcal}$. It is important to remember that this discussion of energy expenditure is dependent on the frequency components associated with monitored behaviors as discussed above, and that it presumes the perspective of a physicist rather than a biologist. Metabolic factors are not presently considered.

\section{BETWEEN-UNIT VARIABILITY}

All the preceding analyses were conducted by repeatedly studying a single actigraph. This section evaluates comparability across devices. Figure 8 is a composite of 40 calibration curves for 40 different Model 7164 activity monitors. The data for this figure were obtained by submitting each actigraph to the spinner described above.

The results show consistent uniformity of response from near zero up to $5 \mathrm{~Hz}$, with somewhat greater dispersion thereafter. The shape of this curve reflects several operating characteristics of the actigraph described above. The

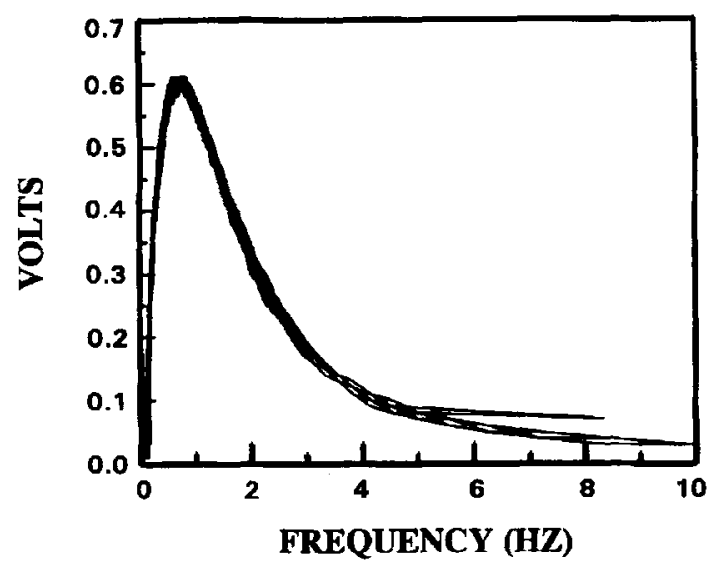

Figure 8. Superimposed calibration curves for $\mathbf{4 0}$ actigraphs. 
actigraph is maximally sensitive to $0.75-\mathrm{Hz}$ activity components. The analog filters set the half-amplitude frequencies at 0.21 and $2.28 \mathrm{~Hz}$ with outer limits of 0.1 and $3.6 \mathrm{~Hz}$. Since frequency components above $3.6 \mathrm{~Hz}$ are immaterial, coherence among devices up to $5 \mathrm{~Hz}$ constitutes excellent between-device agreement.

\section{HUMAN DATA}

Tryon (1991a, pp. 8-14) distinguished between instrument reliability and clinical repeatability. Activity instrument reliability requires that the source of motion be highly controlled, so that nearly all variability can be attributed to the device. This can only be accomplished under laboratory conditions. Attempting to document either the reliability or the validity of any activity monitor by attaching it to subjects and instructing them to behave in prescribed ways always underestimates the reliability and validity of the device to the extent that the human test subjects fail to execute exactly the same movements in time and space. We were careful to control for factors altering the decay of a 5 - $\mathrm{ft}$ pendulum moving about a single joint. Human behavior entails the use of multiple joints and connecting muscles, providing additional sources of error. Biomechanical analyses of gait (Cavagna \& Margaria, 1966; Cavagna, Saibene, \& Margaria, 1963; Liberson, 1965; Morris, 1973; Saunders, Inman, \& Eberhart, 1953) reveal that normal walking causes the waist to rise and fall as well as move left and right in a spiral pattern. These movements, and the impact of the foot meeting the floor, give rise to complex patterns of acceleration that cannot be reproduced with the same degree of precision as a decaying pendulum and the spinning calibration device. The arms and legs allow even more complex motions. Physical differences in the build of different subjects produce different absolute values of accelerations. All this variation will erroneously be attributed to device unreliability and invalidity if human subjects are used to evaluate the operating characteristics of activity monitors.

Clinical repeatability pertains to the conditions under which replicable activity measures can be obtained from subjects despite all the sources of variation that require instrument reliability and validity to be evaluated in the laboratory. The more variable behavior is, the longer it must be measured to produce a mean or variance that will agree with a second assessment of equal duration to within a stated tolerance. By instructing subjects to perform stereotyped behaviors under controlled conditions, one can evaluate the repeatability of activity measurements under "ideal" conditions.

Patterson et al. (1993) demonstrated that repeatable activity measurements could be obtained from wrist-worn actigraphs (Ambulatory Monitoring, Ardsley, NY) capable of discriminating active (walking, running, stair climbing, and knee bending) from passive (reading, typing, playing video games, performing mental arithmetic) behavior in 15 healthy adults. Their second study revealed that activity data collected during a second trial corrre- lated $[r(13)=.98, p<.0001]$ with data collected during the first trial.

Janz (1994) used a different model of CSA actigraph than the one evaluated here, but similar to the Ambulatory Monitoring actigraph used by Patterson et al. (1993), to measure the activity of 31 children ( 16 boys and 15 girls) 7-15 years old for 3 days while concurrently measuring their heart rate (Polar Vantage XL; Polar Electro, Kempele, Finland). The mean correlation between measures was reported to be $r=.69$.

Melanson and Freedson (1995) evaluated the validity of the same make and model of CSA actigraph described above to estimate caloric expenditure. Fifteen male and 13 female college students wore a CSA actigraph on their nonpreferred wrist, waist, and ankle while walking slowly $(4.8 \mathrm{~km} / \mathrm{h})$, walking fast $(6.4 \mathrm{~km} / \mathrm{h})$, and jogging $(8.1 \mathrm{~km} / \mathrm{h})$ for $8 \mathrm{~min}$ on a motorized treadmill on three occasions. The three grade conditions of $0 \%, 3 \%$, and $6 \%$ were separated by a 6 -min rest period. Oxygen consumption was measured with open-circuit spirometry. Regression analysis revealed that kilocalories/minute were highly predictable from hip and wrist activity measurements plus body mass in kilograms $(R=.95)$.

\section{DISCUSSION}

The engineering specifications, calibration data, and between-unit evaluation presented above certify that the CSA Model 7164 instrument is a reliable and valid activity monitor for measuring acceleration forces of \pm 0.05 to $2.13 \mathrm{~g}$ within a passband of $0.1-3.6 \mathrm{~Hz}$ in increments of 1.664 milli-g. These data cannot presently be transformed into average units of kinetic energy, because concurrent frequency information is not recorded. This limitation will be corrected in future models. Previously published data indicate that this actigraph discriminates human activities varying in caloric expenditure.

The Model 7164 sums and stores A/D bit count deviations over a user-defined epoch. Choice of a 1-min epoch allows continuous recording for 22 complete days, which will often constitute an adequate behavioral sample. This technology enables the acquisition of high-quality data for answering questions about infant temperament, early childhood personality, extensions into adult extraversion, behavioral differences between externalizing and internalizing disorders of childhood, sleep disorders, and contributions to DSM-IV psychiatric diagnosis in a cost effective manner. Questions of behavioral variability can also be objectively addressed. The ability to discriminate 128 levels of movement magnitude enables one to measure activity intensity. This may be especially important when one is attempting to quantify fatigue, where lowintensity activity may characterize most activities.

Activity has been analyzed from the perspective of behavioral physics. These results can be used to approximate the metabolic costs associated with movement, but not of maintaining static posture, especially when one is holding heavy objects, because of the absence of movement. 
Table 5

Three Replications on the Spinner for Actigraph Number 52. These Data Were Used to Create Figure 7

\begin{tabular}{|c|c|c|c|c|c|}
\hline \multicolumn{2}{|c|}{ First Run } & \multicolumn{2}{|c|}{ Second Run } & \multicolumn{2}{|c|}{ Third Run } \\
\hline $\mathrm{Hz}$ & $1 \mathrm{~g}$ Volts & $\mathrm{Hz}$ & $1 \mathrm{~g}$ Volts & $\mathrm{Hz}$ & $\lg$ Volts \\
\hline 0.10 & 0.09 & 0.00 & 0.01 & 0.12 & 0.16 \\
\hline 0.12 & 0.13 & 0.08 & 0.10 & 0.14 & 0.19 \\
\hline 0.16 & 0.19 & 0.10 & 0.12 & 0.16 & 0.20 \\
\hline 0.18 & 0.24 & 0.12 & 0.13 & 0.18 & 0.25 \\
\hline 0.20 & 0.27 & 0.16 & 0.22 & 0.20 & 0.28 \\
\hline 0.22 & 0.31 & 0.18 & 0.25 & 0.22 & 0.33 \\
\hline 0.24 & 0.34 & 0.20 & 0.27 & 0.24 & 0.36 \\
\hline 0.26 & 0.37 & 0.22 & 0.32 & 0.26 & 0.38 \\
\hline 0.28 & 0.40 & 0.24 & 0.34 & 0.28 & 0.41 \\
\hline 0.30 & 0.42 & 0.28 & 0.40 & 0.30 & 0.43 \\
\hline 0.34 & 0.45 & 0.30 & 0.43 & 0.32 & 0.46 \\
\hline 0.36 & 0.47 & 0.32 & 0.45 & 0.36 & 0.49 \\
\hline 0.38 & 0.49 & 0.34 & 0.47 & 0.38 & 0.50 \\
\hline 0.40 & 0.51 & 0.36 & 0.48 & 0.40 & 0.52 \\
\hline 0.42 & 0.51 & 0.38 & 0.50 & 0.42 & 0.53 \\
\hline 0.44 & 0.54 & 0.40 & 0.51 & 0.44 & 0.54 \\
\hline 0.46 & 0.54 & 0.42 & 0.52 & 0.46 & 0.54 \\
\hline 0.48 & 0.55 & 0.44 & 0.54 & 0.48 & 0.56 \\
\hline 0.50 & 0.56 & 0.46 & 0.55 & 0.50 & 0.57 \\
\hline 0.52 & 0.57 & 0.48 & 0.55 & 0.52 & 0.57 \\
\hline 0.54 & 0.57 & 0.50 & 0.56 & 0.54 & 0.58 \\
\hline 0.56 & 0.58 & 0.52 & 0.57 & 0.56 & 0.59 \\
\hline 0.58 & 0.58 & 0.56 & 0.58 & 0.60 & 0.59 \\
\hline 0.60 & 0.59 & 0.60 & 0.59 & 0.64 & 0.59 \\
\hline 0.62 & 0.59 & 0.62 & 0.59 & 0.66 & 0.60 \\
\hline 0.64 & 0.59 & 0.64 & 0.59 & 0.68 & 0.59 \\
\hline 0.66 & 0.59 & 0.66 & 0.59 & 0.70 & 0.60 \\
\hline 0.68 & 0.59 & 0.70 & 0.60 & 0.74 & 0.60 \\
\hline 0.70 & 0.60 & 0.72 & 0.60 & 0.76 & 0.60 \\
\hline 0.72 & 0.59 & 0.74 & 0.60 & 0.80 & 0.60 \\
\hline 0.74 & 0.59 & 0.76 & 0.60 & 0.82 & 0.59 \\
\hline 0.76 & 0.59 & 0.80 & 0.59 & 0.84 & 0.59 \\
\hline 0.78 & 0.59 & 0.82 & 0.59 & 0.86 & 0.59 \\
\hline 0.80 & 0.59 & 0.84 & 0.59 & 0.88 & 0.59 \\
\hline 0.84 & 0.59 & 0.88 & 0.59 & 0.90 & 0.59 \\
\hline 0.86 & 0.59 & 0.90 & 0.58 & 0.92 & 0.58 \\
\hline 0.88 & 0.58 & 0.94 & 0.58 & 0.94 & 0.58 \\
\hline 0.90 & 0.58 & 0.96 & 0.57 & 0.98 & 0.57 \\
\hline 0.92 & 0.58 & 0.98 & 0.57 & 1.02 & 0.57 \\
\hline 0.96 & 0.58 & 1.00 & 0.57 & 1.04 & 0.56 \\
\hline 0.98 & 0.57 & 1.02 & 0.56 & 1.06 & 0.56 \\
\hline 1.00 & 0.56 & 1.04 & 0.56 & 1.08 & 0.55 \\
\hline 1.04 & 0.56 & 1.08 & 0.55 & 1.12 & 0.55 \\
\hline 1.06 & 0.55 & 1.12 & 0.55 & 1.14 & 0.54 \\
\hline 1.08 & 0.55 & 1.14 & 0.54 & 1.16 & 0.53 \\
\hline 1.12 & 0.55 & 1.16 & 0.54 & 1.20 & 0.53 \\
\hline 1.14 & 0.54 & 1.20 & 0.53 & 1.24 & 0.52 \\
\hline 1.16 & 0.53 & 1.22 & 0.52 & 1.28 & 0.51 \\
\hline 1.20 & 0.53 & 1.24 & 0.52 & 1.32 & 0.50 \\
\hline 1.22 & 0.52 & 1.28 & 0.51 & 1.38 & 0.49 \\
\hline 1.24 & 0.52 & 1.32 & 0.49 & 1.42 & 0.47 \\
\hline 1.28 & 0.50 & 1.36 & 0.50 & 1.48 & 0.46 \\
\hline 1.36 & 0.50 & 1.38 & 0.49 & 1.52 & 0.46 \\
\hline 1.38 & 0.48 & 1.42 & 0.47 & 1.56 & 0.44 \\
\hline 1.42 & 0.47 & 1.48 & 0.46 & 1.62 & 0.43 \\
\hline 1.48 & 0.46 & 1.52 & 0.45 & 1.66 & 0.42 \\
\hline 1.52 & 0.45 & 1.56 & 0.44 & 1.72 & 0.40 \\
\hline 1.56 & 0.44 & 1.62 & 0.43 & 1.78 & 0.39 \\
\hline 1.62 & 0.43 & 1.66 & 0.41 & 1.86 & 0.38 \\
\hline 1.66 & 0.42 & 1.72 & 0.40 & 1.92 & 0.36 \\
\hline 1.72 & 0.41 & 1.78 & 0.39 & 2.00 & 0.35 \\
\hline 1.78 & 0.40 & 1.86 & 0.38 & 2.08 & 0.33 \\
\hline 1.86 & 0.38 & 1.92 & 0.36 & 2.18 & 0.32 \\
\hline 1.92 & 0.36 & 2.00 & 0.35 & 2.28 & 0.30 \\
\hline 2.00 & 0.35 & 2.08 & 0.33 & 2.38 & 0.28 \\
\hline
\end{tabular}

Table 5 (Continued)

\begin{tabular}{|c|c|c|c|c|c|}
\hline \multicolumn{2}{|c|}{ First Run } & \multicolumn{2}{|c|}{ Second Run } & \multicolumn{2}{|c|}{ Third Run } \\
\hline $\mathrm{Hz}$ & $1 \mathrm{~g}$ Volts & $\mathrm{Hz}$ & $1 \mathrm{~g}$ Volts & $\mathrm{Hz}$ & $1 \mathrm{~g}$ Volts \\
\hline 2.08 & 0.34 & 2.18 & 0.32 & 2.50 & 0.26 \\
\hline 2.18 & 0.31 & 2.28 & 0.29 & 2.64 & 0.24 \\
\hline 2.28 & 0.29 & 2.38 & 0.28 & 2.78 & 0.22 \\
\hline 2.38 & 0.28 & 2.50 & 0.26 & 2.94 & 0.21 \\
\hline 2.50 & 0.26 & 2.64 & 0.25 & 3.12 & 0.19 \\
\hline 2.64 & 0.24 & 2.78 & 0.23 & 3.34 & 0.17 \\
\hline 2.78 & 0.22 & 2.94 & 0.20 & 3.58 & 0.15 \\
\hline 2.94 & 0.21 & 3.12 & 0.19 & 3.84 & 0.13 \\
\hline 3.12 & 0.19 & 3.34 & 0.16 & 4.16 & 0.11 \\
\hline 3.34 & 0.17 & 3.58 & 0.15 & 4.54 & 0.10 \\
\hline 3.58 & 0.14 & 3.84 & 0.13 & 5.00 & 0.08 \\
\hline 3.84 & 0.13 & 4.16 & 0.11 & 5.56 & 0.07 \\
\hline 4.16 & 0.11 & 4.54 & 0.10 & 6.24 & 0.06 \\
\hline 4.54 & 0.10 & 5.00 & 0.08 & 7.14 & 0.05 \\
\hline 5.00 & 0.08 & 5.56 & 0.07 & 8.34 & 0.04 \\
\hline 5.56 & 0.06 & 6.24 & 0.06 & 10.00 & 0.03 \\
\hline 6.24 & 0.06 & 7.14 & 0.05 & & \\
\hline 7.14 & 0.05 & 8.34 & 0.04 & & \\
\hline 8.34 & 0.04 & 10.00 & 0.03 & & \\
\hline 10.00 & 0.03 & & & & \\
\hline
\end{tabular}

Energy expenditure is defined from the perspective of the physicist rather than biologist. The problem is symmetric, in that heart rate, oxygen consumption, and other physiological measures of activity can be elevated by stress, anxiety, and certain medications in the absence of observable activity. Therefore, one cannot validate one approach against the other but must evaluate each against its own theoretical basis. This report evaluates the CSA actigraph against a physical understanding of motion and energy expenditure.

CSA actigraphs are reliable and valid when used to monitor movements within the design parameters (up to $2.13 \mathrm{~g}$ and between 0.1 and $3.6 \mathrm{~Hz}$ ). Reliability and validity will be compromised if one attempts to track movements that exceed these design specifications. Hence, the same device can be valid or invalid, depending on the application to which it is put. It is therefore unreasonable to discuss the validity of this, or any, activity monitor apart from its intended application. Prior to data collection, investigators should therefore familiarize themselves with both the design specifications of the activity monitor that they intend to use and the acceleration forces associated with the behaviors that they intend to monitor.

It will usually be impracticable to continuously measure the $g$ forces associated with behaviors like those to be monitored with an accelerometer and an analog tape recorder. Fortunately, such detailed information is probably not necessary in most instances. The least and most vigorous behaviors are especially informative. The concern is whether the activity monitor will detect the least vigorous behavior and whether it will saturate before the maximum acceleration associated with the most vigorous behavior has been reached. This information can be obtained by attaching the activity monitor to subjects and having them engage in representative behaviors. The ques- 
tion of whether the activity monitor is sensitive to the least vigorous behavior is answered by instructing subjects to emit only such behaviors, downloading the data, and determining whether any activity counts resulted. One cannot answer the saturation question as easily, because it depends on the maximum acceleration encountered, the rate with which acceleration values change, and the duration of extreme values. It is possible for the maximum acceleration emitted by a subject to momentarily exceed the instrument's upper linear bound and not produce a fully proportional activity measurement over the standard 1-min epoch. Reducing the epoch length to 10,5 , or $1 \mathrm{sec}$ will help determine whether, and for approximately how long, the dynamic range has been exceeded.

In conclusion, technology now enables reliable and valid activity-intensity proportional measurement every minute of the day and night for 22 continuous 24 -h periods and for weeks or months through repeated assessments. The ability to obtain comprehensive, cost-effective, highquality activity measurements directly from naturalistic conditions makes it no longer necessary to generalize from brief laboratory or clinic observations and/or ratings to natural behavior in various environments. Extensive behavioral samples can be obtained directly.

Collection of data points at known, and constant, intervals strongly satisfies data requirements for timeseries analysis. Collecting consecutive $24-\mathrm{h}$ behavioral samples enables chronobiological analyses of circadian rhythm. Instrumented activity measurement provides data that are not contaminated by shared method variance when correlated with clinical ratings of adjustment, aggression, and so forth (Schachar, Sandberg, \& Rutter, 1986). In sum, current activity measurement technology offers many advantages to clinicians and researchers.

\section{REFERENCES}

AChenbach, T. M., \& Edelbrock, C. S. (1978). The classification of child psychopathology: A review and analysis of empirical efforts. Psychological Bulletin, 85, 1275-1301.

Achenbach, T. M., Verhulst, F. C., Baron, G. D., \& Althaus, M. (1987). A comparison of syndromes derived from the Child Behavior Checklist for American and Dutch boys aged 6-11 and 12-16. Journal of Child Psychology \& Psychiatry \& Allied Disciplines, 28 , 437-453.

Buss, A. H., \& Plomin, R. (1975). A temperament theory of personality development. New York: Wiley.

Buss, A. H., \& Plomin, R. (1984). Temperament: Early developing personality traits. Hillsdale, NJ: Erlbaum.

Cavagna, G. A., \& Margaria, R. (1966). Mechanics of walking. Journal of Applied Physiology, 21, 271-278.

Cavagna, G. A., Saibene, F. P., \& Margaria, R. (1963). External work in walking. Journal of Physiology, 18, 1-9.

Costa, P. T., JR., \& MCCraE, R. R. (1980). Still stable after all these years: Personality as a key to some issues in adulthood and old age. In P. B. Baltes \& O. G. Brim (Eds.), Life-span development and behavior (Vol. 3, pp. 65-102). New York: Academic Press.

Costa, P. T., JR., \& McCrae, R. R. (1992). Revised NEO Personality Inventory (NEO PI-R) and NEO Five-Factor Inventory (NEO-FFI): Professional manual. Odessa, FL: Psychological Assessment Resources, Inc.

Costa, P. T., JR., \& MCCrae, R. R. (1994). Stability and change in personality from adolescence through adulthood. In C. F. Halverson, Jr., G. A. Kohnstamm, \& R. P. Martin (Eds.), The developing structure of temperament and personality from infancy to adulthood (pp. 139 150). Hillsdale, NJ: Erlbaum.

Costa, P. T., JR., \& McCraE, R. R. (1995). Solid ground in the wetlands of personality: A reply to Block. Psychological Bulletin, 117, 216-220.

Digman, J. (1990). Personality structure. Emergence of the five factor model. Annual Review of Psychology, 41, 417-440.

Digman, J. (1994). Child personality and temperament: Does the fivefactor model embrace both domains? In C. F. Halverson, Jr., G. A. Kohnstamm, \& R. P. Martin (Eds.), The developing structure of temperament and personality from infancy to adulthood (pp. 323-338). Hillsdale, NJ: Erlbaum.

EYSENCK, H. J. (1994). The big five or giant three: Criteria for a paradigm. In C. F. Halverson, Jr., G. A. Kohnstamm, \& R. P. Martin (Eds.), The developing structure of temperament and personality from infancy to adulthood (pp. 37-51). Hillsdale, NJ: Erlbaum.

FrEEDSON, P. S. (1991). Electronic motion sensors and heart rate as measures of physical activity in children. Journal of School Health, 61, 220-223.

Gerhardsson, M., Norell, S. E., Kiviranta, H., Pedersen, N. L., \& AнLвом, A. (1986). Sedentary jobs and colon cancer. American Journal of Epidemiology, 123, 775-780.

GoldBerG, L. R., \& RosolaCK, T. K. (1994). The big five factor structure as an integrative framework: An empirical comparison with Eysenck's P-E-N model. In C. F. Halverson, Jr., G. A. Kohnstamm, \& R. P. Martin (Eds.), The developing structure of temperament and personality from infancy to adulthood (pp. 7-35). Hillsdale, NJ: Erlbaum.

Goldsmith, H. H., Buss, A. H., Plomin, R., Rothbart, M. K., Thomas, A., Chess, S., Hinde, R. A., \& McCall, R. B. (1987). Roundtable: What is temperament? Four approaches. Child Development, 58, 505-529.

Halverson, C. F., Jr., Kohnstamm, G. A., \& Martin, R. P. (1994). The developing structure of temperament and personality from infancy to adulthood. Hillsdale, NJ: Erlbaum.

HINSHAW, S. P. (1987). On the distinction between attentional deficit/ hyperactivity and conduct problems/aggression in child psychopathology. Psychological Bulletin, 101, 443-463.

HINSHAW, S. P. (1992). Externalizing behavior problems and academic under achievement in childhood and adolescence: Causal relationships and underlying mechanisms. Psychological Bulletin, 111, 127-155.

Hubert, N. C., Wachs, T. D., Peters-Martin, P., \& Gandour, M. J. (1982). The study of early temperament: Measurement and conceptual issues. Child Development, 53, 571-600.

JANZ, K. F. (1994). Validation of the CSA accelerometer for assessing children's physical activity. Medicine \& Science in Sports \& Exercise, 26, 369-375.

KaGAN, J., \& SNIDMAN, N. (1991). Temperamental factors in human development. American Psychologist, 46, 856-862.

LaPorte, R. E., Montoye, H. J., \& CAspersen, C. J. (1985). Assessment of physical activity in epidemiologic research: Problems and prospects. Public Health Reports, 100, 131-146.

LiBERSON, W. T. (1965). Biomechanics of gait: A method of study. Archives of Physical Medicine, 46, 37-48.

MASON, D. J., \& REDEKER, N. (1993). Measurement of activity. Nursing Research, 42, 87-92.

MCCraE, R. R., \& John, O. P. (1992). An introduction to the five-factor model and its applications. Journal of Personality, 60, 175-215.

Melanson, E. L., JR., \& Freedson, P. S. (1995). Validity of the Computer Science Associates (CSA) activity monitor. Medicine \& Science in Sports \& Exercise, 27, 934-940.

Melanson, E. L., JR., \& Freedson, P. S. (1996). Physical activity assessment: A review of methods. Critical Reviews in Food Science \& Nutrition, 36, 385-396.

MONTOYE, H. J., \& TAYLOR, H. L. (1984). Measurement of physical activity in population studies: A review. Human Biology, 56, 195-216.

MoRrIs, J. R. W. (1973). Accelerometry-A technique for the measurement of human body movements. Journal of Biomechanics, 6, 729-736.

Ozer, D. J., \& ReISE, S. P. (1994). Personality assessment. Annual Review of Psychology, 45, 357-388.

Patterson, S. M., Krantz, D. S., Montgomery, L. C., Deuster, P. A., Hedges, S. M., \& Nebel, L. E. (1993). Automated physical ac- 
tivity monitoring: Validation and comparison with physiological and self-report measures. Psychophysiology, 30, 296-305.

Redeker, N. S., Mason, D. J., WykPISZ, E. M., \& Glica, B. (1995). Women's activity pattern over six months after coronary artery bypass. Heart \& Lung, 24, 502-511.

Redeker, N. S., MASON, D. J., WykPISZ, E. M., Glica, B., \& Miner, C. (1994). First post-operative week activity patterns in women after coronary artery bypass. Nursing Research, 43, 168-173.

Saunders, J. B., InMaN, V. T., \& EberharT, H. D. (1953). The major determinants in normal and pathological gait. Journal of Bone \& Joint Surgery, 35A, 543-558.

SCHACHAR, R., SANDBERG, S., \& RUTTER, M. (1986). Agreement between teachers' ratings and observations of hyperactivity, inattentiveness, and defiance. Journal of Abnormal Child Psychology, 14, 331-345.

Siscovick, D. S., LAPorTe, R. E., \& NewMAN, J. M. (1985). The diseasespecific benefits and risks of physical activity and exercise. Public Health Reports, 100, 180-188.

Slattery, M. L., Schumacher, M. C., Smith, K. R., West, D. W., \& ABd-Elghany, N. (1988). Physical activity, diet, and risk of colon cancer in Utah. American Journal of Epidemiology, 128, 989-999.

Thomas, A., \& Chess, S. (1977). Temperament and development. New York: Brunner/Mazel.

Thomas, A., Chess, S., \& Birch, H. G. (1968). Temperament and behavior disorders in children. New York: New York University Press.

Thomas, A., Chess, S., Birch, H. [G.], Hertzig, M., \& Korn, S.
(1963). Behavioral individuality in early childhood. New York: New York University Press.

TRYON, W. W. (1985). The measurement of human activity. In W. W. Tryon (Ed.), Behavioral assessment in behavioral medicine (pp. 200-256). New York: Springer-Verlag.

Tryon, W. W. (1991a). Activity measurement in psychology and medicine. New York: Plenum.

TRYON, W. W. (1991b). Motoric assessment and DSM-III-R. In M. Hersen \& S. M. Turner (Eds.), Adult psychopathology and diagnosis (pp. 413-440). New York: Wiley.

TRYON, W. W. (1993). The role of motor excess and instrumented activity measurement in attention-deficit hyperactivity disorder. $\mathrm{Be}$ havior Modification, 17, 371-406.

TRYON, W. W. (in press-a). Motor activity and DSM-IV. In S. Turner \& M. Hersen (Eds.), Adult psychopathology and diagnosis (3rd. ed.). New York: Wiley.

TRYON, W. W. (in press-b). Physical activity. In M. Hersen \& B. B. Van Hasselt (Eds.), Handbook of clinical geropsychology. New York: Plenum.

ZuCKerman, M. (1991). Psychobiology of personality. Cambridge: Cambridge University Press.

(Manuscript received February 24, 1995; revision accepted for publication June 9, 1995.) 